OPEN ACCESS

Edited by:

Junchao Zheng,

Central South University, China

Reviewed by:

Haisheng Fang,

Kunming University of Science and

Technology, China

Chaoyi Chen,

Guizhou University, China

Ying Hong Yang,

Northeastern University, China

*Correspondence:

Zhao Fang

fangzhao@xauat.edu.cn

Specialty section:

This article was submitted to Electrochemistry,

a section of the journal

Frontiers in Chemistry

Received: 21 April 2019 Accepted: 26 June 2019

Published: 18 July 2019

Citation:

Yu J, Gao N, Peng J, Ma N, Liu X,

Shen C, Xie K and Fang Z (2019) Concentrated LiODFB Electrolyte for

Lithium Metal Batteries.

Front. Chem. 7:494.

doi: 10.3389/fchem.2019.00494

\section{Concentrated LiODFB Electrolyte for Lithium Metal Batteries}

\author{
Juan $\mathrm{Yu}^{1}$, Na Gao ${ }^{1}$, Jiaxin Peng ${ }^{1}$, Nani Ma ${ }^{1}$, Xiaoyan $\mathrm{Liu}^{2}$, Chao Shen ${ }^{2}, \mathrm{Keyu} \mathrm{Xie}^{2}$ and \\ Zhao Fang $^{1 *}$ \\ 'School of Metallurgical Engineering, Xi'an University of Architecture and Technology, Xi'an, China, ${ }^{2}$ State Key Laboratory of \\ Solidification Processing, Center for Nano Energy Materials, School of Materials Science and Engineering, Northwestern \\ Polytechnical University and Shaanxi Joint Laboratory of Graphene (NPU), Xi'an, China
}

Nowadays, lithium (Li) metal batteries arouse widespread concerns due to its ultrahigh specific capacity $\left(3,860 \mathrm{mAh} \mathrm{g}^{-1}\right)$. However, the growth of Li dendrites has always limited their industrial development. In this paper, the use of concentrated electrolyte with lithium difluoro(oxalate)borate (LiODFB) salt in 1, 2-dimethoxyethane (DME) enables the good cycling of a Li metal anode at high Coulombic efficiency (up to 98.1\%) without dendrite growth. Furthermore, a Li/Li cell can be cycled at $1 \mathrm{~mA} \mathrm{~cm}{ }^{-2}$ for over 3,000 h. Besides, compared to conventional $\mathrm{LiPF}_{6}$-carbonate electrolyte, $\mathrm{Li} / \mathrm{LiFePO}{ }_{4}$ cells with $4 \mathrm{M}$ LiODFB-DME exhibit superior electrochemical performances, especially at high temperature $\left(65^{\circ} \mathrm{C}\right)$. These outstanding performances can be certified to the increased availability of $\mathrm{Li}^{+}$concentration and the merits of LiODFB salt. We believe that the concentrated LiODFB electrolyte is help to enable practical applications for Li metal anode in rechargeable batteries.

Keywords: Li-metal batteries, concentrated electrolyte, LiODFB, high temperature, dendrites free

\section{INTRODUCTION}

In the past several decades, Li-ion batteries have played successful role in the consumable electronic device market (Etacheri et al., 2011; Goodenough and Kim, 2014). However, the limited specific capacity of the graphite anode limits its wider applications. The theoretical capacity of graphite is only $372 \mathrm{mAh} \mathrm{g}^{-1}$, so it is difficult to achieve the urgent need for high-energy density batteries to adapt to the electrical miniaturization trend (Placke et al., 2017). Based on this reason, the Li metal batteries (LMBs) have attracted wide attention because of its high theoretical specific capacity of $3,860 \mathrm{mAh} \mathrm{g}^{-1}$, small density of $0.534 \mathrm{~g} \mathrm{~cm}^{-3}$ and the relatively negative electrochemical potential $\left(-3.040 \mathrm{~V} \mathrm{vs} . \mathrm{Li} / \mathrm{Li}^{+}\right)$(Zhamu et al., 2012). However, the shortcomings of LMBs are also a headache, such as unsatisfied Coulombic efficiency (CE) and dendritic Li growth. In addition, poor cycle performance caused by Li dendrites seriously affected the commercial development of LMBs.

Significant efforts have been made in the past to solve these problems, including prepare the current collector (Yang et al., 2015; Liang et al., 2016; Zhang et al., 2016; Shi et al., 2017), ex-situ protective coating of Li anode (Thompson et al., 2011; Kozen et al., 2015), introduce the battery intermediate protective layer (Liang et al., 2015; Wu et al., 2015; Cheng et al., 2016; Xie et al., 2016, 2017), and electrolyte modification. Electrolyte, which occupies $15 \%$ of the weight and about $32 \%$ of the volume of the entire battery, is a key part of a battery (Cekic-Laskovic et al., 2017). Therefore, the study of electrolyte on the development of Li metal secondary battery is of great significance. Meanwhile, compared to other modification methods, electrolyte modification is more convenient and cost-effective. Till now, electrolyte research progress more or less has been made to 
address the dendrite problem. Nevertheless, the compatibility of these electrolytes with cathode has usually been overlooked. For example, some film-forming electrolyte additives, such as vinylene carbonate (VC) and fluoroethylene carbonate (FEC) are helpful to change Li dendrite formation nature (Aurbach et al., 2003; Jung et al., 2013; Webb et al., 2014; Qian et al., 2016; Pritzl et al., 2017; Xu et al., 2017), but the cell's internal resistance is greatly increased, particularly after long-term cycling, and thus limits their wide applications.

Recently, the concept of high concentrated electrolyte gets into the eyes of people. Suo et al. reported a new class of nonaqueous liquid "Solvent-in-Salt" electrolytes and applied them in Li-S batteries. It is demonstrated that the use of "Solventin-Salt" electrolyte inhibits the dissolution of polysulphide and protects metallic $\mathrm{Li}$ anodes against the formation of $\mathrm{Li}$ dendrites, effectively (Suo et al., 2013). A significant breakthrough has been achieved by Qian et al. (2015). They found that, with $4 \mathrm{M}$ lithium bis(fluorosulfonyl)imide (LiFSI) in DME as the electrolyte, a $\mathrm{Li} / \mathrm{Li}$ cell can be cycled at for more than 6,000 cycles. Meanwhile, a $\mathrm{Cu} / \mathrm{Li}$ cell can be cycled for more than 1,000 cycles with an average CE of $98.4 \%$, without dendrite growth. Nevertheless, the compatibility of the electrolyte with the cathode materials has not been investigated.

Generally, LiFSI has inherent characteristics of corrosion of aluminum ( $\mathrm{Al}$ ) foil and other metal parts in the battery (Abouimrane et al., 2009; Li et al., 2011). To solve this problem, Park et al. found that lithium borate salts are the ideal additives as corrosion inhibitors in LiFSI electrolytes. The inhibition ability of $\mathrm{Al}$ is revealed to be in the following order: lithium oxalyldifluoroborate (LiODFB) > lithium tetrafluoroborate $\left(\mathrm{LiBF}_{4}\right)>$ lithium hexafluorophosphate $\left(\mathrm{LiPF}_{6}\right)>$ lithium bis(oxalato)borate (LiBOB) (Park et al., 2015). Noticed that LiODFB is considered to combine the half structures of $\mathrm{LiBOB}$ and $\mathrm{LiBF}_{4}$, and thus, it combines the advantages of $\mathrm{LiBOB}$ and $\mathrm{LiBF}_{4}$ (Zhang, 2006). The main advantages of this salt is given by low viscosity, high ionic conductivity, good film-forming, high temperature performance, good compatibility with the positive electrode, passivation of $\mathrm{Li}$ foil and so on (Liu et al., 2007; Zugmann et al., 2011; Wu et al., 2012; Zhou et al., 2012).

However, it should be noted that previous studies (Zhang et al., 2010; Li et al., 2015; Zhou et al., 2016; Bian et al., 2017) only focused on the effect of LiODFB as an additive or auxiliary salt on battery performances. So far, high concentration of LiODFB single salt in ether solvent for LMBs has not been systematically reported before (Zhang et al., 2015; Poyraz et al., 2019; Yamada et al., 2019). Thus, in this work, we first employed the concentrated electrolyte based on LiODFB in LMBs. Due to its increased availability of $\mathrm{Li}^{+}$concentration, a highly uniform and stable solid electrolyte interface (SEI) film was formed on Li metal anode without any dendrite. Meanwhile, the compatibility of concentrated LiODFB electrolyte and $\mathrm{LiFePO}_{4}$ cathode was first investigated by the electrochemical performance of $\mathrm{Li} / \mathrm{LiFePO}_{4}$ cell at room temperature and high temperature. We found that $4 \mathrm{M}$ LiODFB-DME significantly improve electrochemical performance of $\mathrm{LiFePO}_{4}$ cathode, compared to $\mathrm{LiPF}_{6}$ electrolyte. In particular, at high temperature, the improvement is much greater. In a sense, our work shows concentrated LiODFB electrolyte may have great potential for LMBs.

\section{EXPERIMENTAL SECTION}

\section{Electrolytes and Electrode Preparation}

The $\mathrm{LiPF}_{6}$ dissolved in ethylene carbonate (EC), ethyl methyl carbonate (EMC), dimethyl carbonate (DMC) with a volume ratio of 1:1:1 was formed 1.0 $\mathrm{M} \mathrm{LiPF}_{6}$ electrolyte and used as the blank electrolyte. LiODFB was purchased from Suzhou Fluolyte Co., Ltd., China. LiODFB was dissolved in dimethyl ether (DME) and formed $4 \mathrm{M}$ LiODFB-DME in an Ar-filled glove box $\left(\mathrm{H}_{2} \mathrm{O}<\right.$ $0.1 \mathrm{ppm}, \mathrm{O}_{2}<0.1 \mathrm{ppm}$ ). The electrolytes were stirred to maintain homogeneity, then sealed and stored in the glove box.

The $\mathrm{LiFePO}_{4}$ cathodes were prepared by mixing $80 \mathrm{wt} . \%$ $\mathrm{LiFePO}_{4}$ (Aladdin Co., Ltd. China), 10 wt.\% conductive carbon, and $10 \mathrm{wt} \%$ polyvinylidene (PVDF, Solef) binder in Nmethylpyrrolidone (Kelong, Chengdu). The mixed slurry was coated onto $\mathrm{Al}$ foil and then dried overnight at $110^{\circ} \mathrm{C}$ under vacuum. Disk-shaped electrodes with a diameter of $13 \mathrm{~mm}$ were then punched from the foil (the active material loading was $1.52 \mathrm{mg} \mathrm{cm}^{-2}$ ). CR2016 coin-type cells were assembled in an Arfilled glove box with an $\mathrm{LiFePO}_{4}$ cathode working electrode, a Li foil counter electrode, and a polypropylene (Celgard 2400) separator; $45 \mathrm{~mL}$ of electrolyte was then injected into the cells. Finally, the assembled coin cells were sealed for further tests. $\mathrm{Li} / \mathrm{Li}$ and $\mathrm{Li} / \mathrm{Cu}$ cells with different electrolyte solutions and the above-mentioned separator were assembled for further tests.

\section{Electrochemical Measurements}

The assembled $\mathrm{Li} / \mathrm{LiFePO}_{4}$ coin cells were left to stand for $10 \mathrm{~h}$ before galvanostatic precycling for 200 cycles at $1^{\circ} \mathrm{C}\left(1^{\circ} \mathrm{C}=170 \mathrm{mAh} \mathrm{g}^{-1}\right)$ between 2.5 and $4.2 \mathrm{~V}$ at $25^{\circ} \mathrm{C}$ using the Land battery test system (Wuhan LANHE Electronics, China). Rate-performance tests were conducted by changing the rate $\left(0.2,0.5,1.0,2.0,5.0\right.$, and $\left.0.2^{\circ} \mathrm{C}\right)$ every 10 cycles. Cyclic voltammetry (CV) and electrochemical impedance spectroscopy (EIS) measurements were carried out using a SI-1287 Electrochemical System (Transmission Precision Measurement Company, UK); the CV tests were performed between 2.5 and $4.2 \mathrm{~V}$ at a scan rate of $0.1 \mathrm{mV} \mathrm{s}^{-1}$ and the EIS tests were performed over $100 \mathrm{kHz}$ to $10 \mathrm{mHz}$ range with an amplitude of $10 \mathrm{mV}$ before and after rate tests. Then the assembled cells were galvanostatic precycled at $65^{\circ} \mathrm{C}$.

The current density for the Li metal plating/stripping was set to $1.0 \mathrm{~mA} \mathrm{~cm}{ }^{-2}$ using a Land battery testing station at room temperature. And the deposition time was $1.5 \mathrm{~h}$. The effective area of the $\mathrm{Cu}$ foil for Li deposition was $2.11 \mathrm{~cm}^{2}$. The current density was $1.0 \mathrm{~mA} \mathrm{~cm}^{-2}$ from 0.01 to $1.00 \mathrm{~V}$. $\mathrm{Li} / \mathrm{Li}$ symmetric cells were assembled with $\mathrm{Li}$ metal used as the working and counter electrodes. The batteries were tested in a Tenney JR environmental chamber to ensure the stable temperature during long-term cycling process.

\section{Morphological Characterizations}

Scanning electron microscopy (SEM) (FE-SEM, LEO 1530) was employed to observe the surface topography of the $\mathrm{LiFePO}_{4}$ 


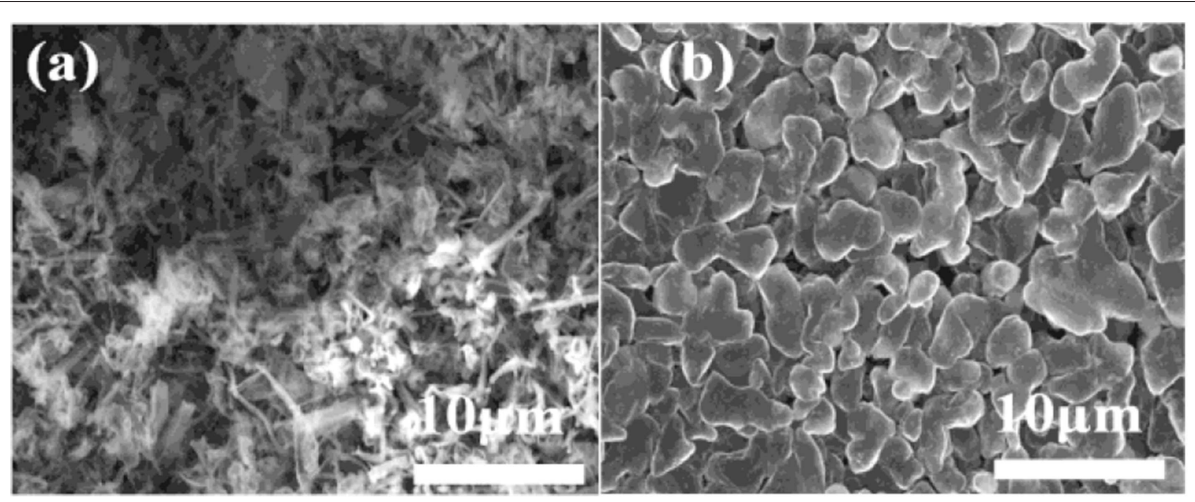

FIGURE 1 | SEM images of the morphologies of Li after plating on Cu substrates in different electrolytes. (a) $1 \mathrm{M}$ LiPF 6 electrolyte; (b) $4 \mathrm{M} \mathrm{LiODFB-DME.} \mathrm{The} \mathrm{current}$ density was $1.0 \mathrm{~mA} \mathrm{~cm}^{-2}$ and the deposition time was $1.5 \mathrm{~h}$.

\section{A}

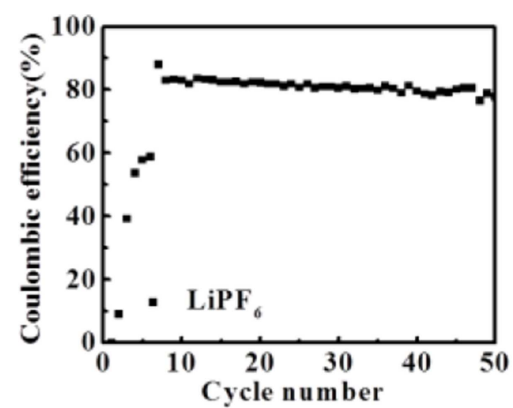

\section{C}

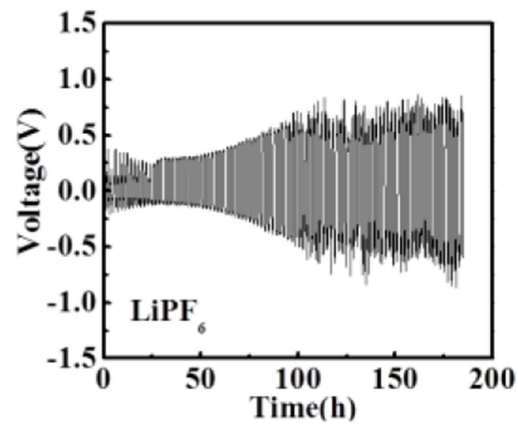

B

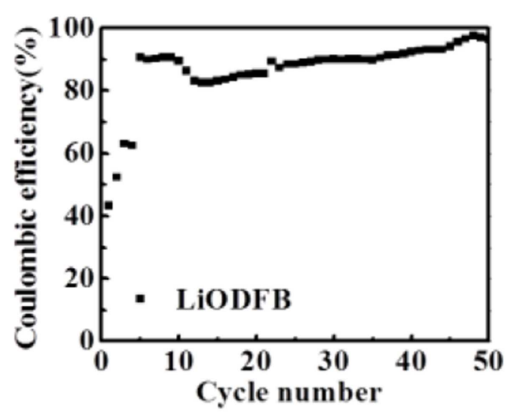

D

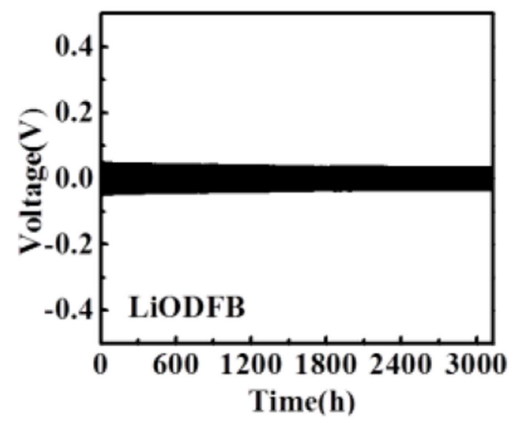

FIGURE 2 | Electrochemical performances of Li/Cu cell $\mathbf{( A , B )}$ and Li/Li cells $(\mathbf{C}, \mathbf{D})$ with two electrolytes at a current density of $1.0 \mathrm{~mA} \mathrm{~cm}{ }^{-2}$. (A,C) the $1 \mathrm{M}$ LiPF 6 electrolyte; (B,D) the 4 M LiODFB-DME.

electrode and $\mathrm{Cu}$ foil at different cycling stages at an accelerating voltage of $5 \mathrm{kV}$. The XPS is tested to further study the composition of SEI film on the Cu foil by the Thermo Electron model K-Alpha surface analysis system. The electrodes retrieved from the cells were thoroughly rinsed in high purity dimethyl carbonate (DMC) solvent for three times and dried under vacuum before transferring to the observation chamber.

\section{EXPERIMENTAL RESULTS}

\section{Li Metal Deposition Morphology}

The morphologies of $\mathrm{Li}$ deposition in both electrolytes are evaluated by using coin-type $\mathrm{Cu} / \mathrm{Li}$ cells. The surface morphologies of Li electrodes cycled after $1.5 \mathrm{~h}$ in different electrolytes is shown in Figure 1. In conventional carbonate based electrolyte, significant dendrite morphology can be observed (Figure 1a). It can easily pierce most conventional separators, causing the direct contact of the positive and negative electrodes to form a short circuit, and lead to serious security risks. On the contrary, Li deposit from the $4 \mathrm{M}$ LiODFBDME electrolyte exhibits smooth solid particle morphology (Figure 1b), which dramatically inhibits the growth of $\mathrm{Li}$ dendrite. Based on the Li metal deposition morphologies, it is reasonable to conclude that the $4 \mathrm{M}$ LiODFB-DME has good negative electrode stability. The SEI film formed in the high concentration electrolyte has high ionic conductivity, which makes a uniform and stable SEI film formed. The uniform and stable SEI film can suppress the growth of Li dendrite. 
A
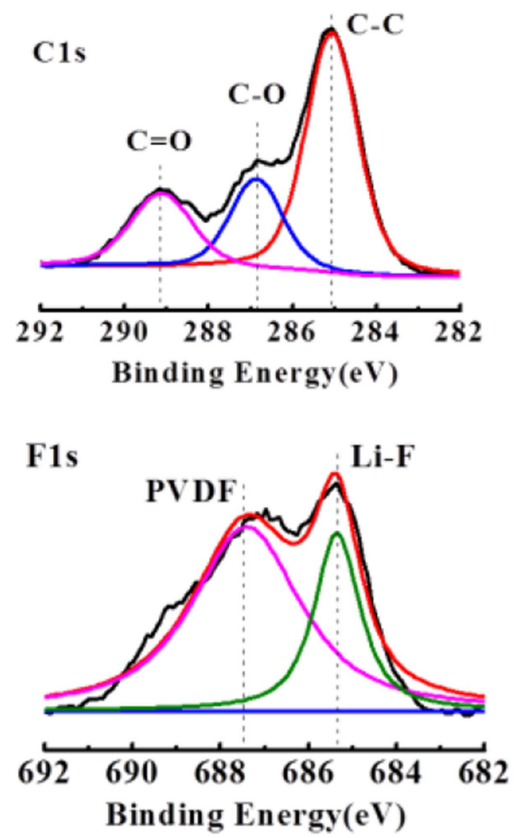

B

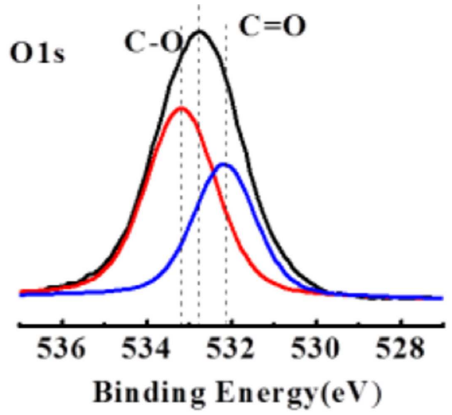

D

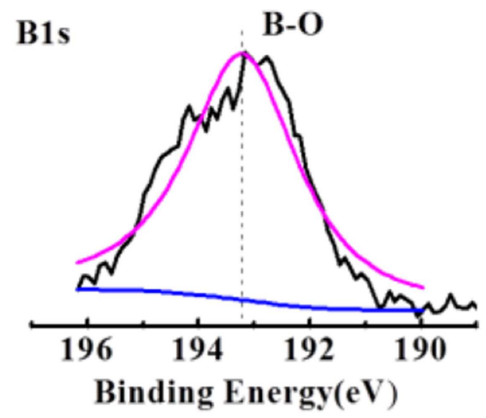

FIGURE 3 | XPS patterns of $(\mathbf{A}, \mathbf{B})$ the cycled Li anodes in the LiODFB electrolyte. (C,D) The cycled electrodes were disassembled from the Li/Li cells after $1.5 \mathrm{~h}$.

A
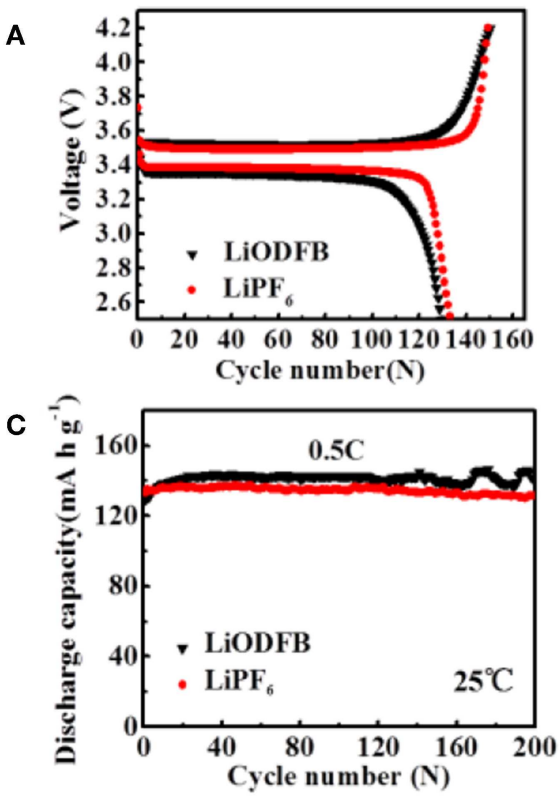

B

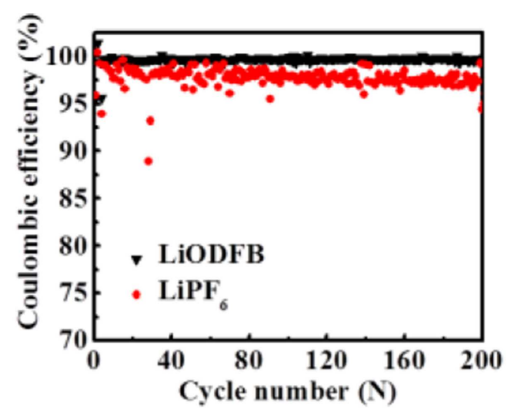

D

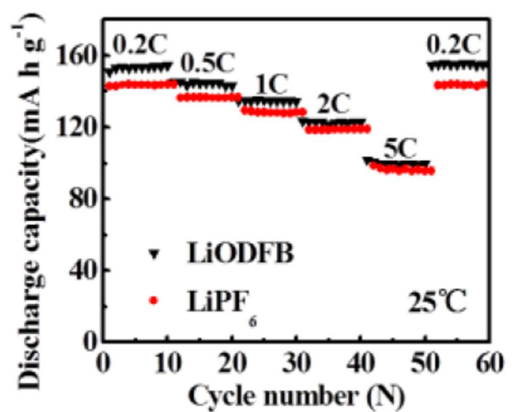

FIGURE 4 | (A) first charge/discharge profiles the Li/LiFePO 4 cells, (B) CE of Li/LiFePO 4 cells; (C,D) cycle performance of Li/LiFePO 4 cells at $25^{\circ} \mathrm{C}$ with $4 \mathrm{M}^{\circ}$ LiODFB-DME and $1 \mathrm{M} \mathrm{LiPF}_{6}$, (D) rate performances of Li/LiFePO 4 cells at $25^{\circ} \mathrm{C}$ with $4 \mathrm{M} \mathrm{LiODFB-DME}$ and $1 \mathrm{M} \mathrm{LiPF}_{6}$.

\section{Li Metal Plating/Stripping Cycling Stability}

The impedance evolution of the coin-type $\mathrm{Cu} / \mathrm{Li}$ cells after $0.5 \mathrm{~h}$ and $1.5 \mathrm{~h}$ are depicted in Figure $\mathbf{S 1}$. In the Nyquist plots, the intercept with the abscissa represents the ohmic resistance, particularly the electrolyte resistance in the cell.
A significant difference in the impedance between the two electrolytes is observed. After 0.5 and $1.5 \mathrm{~h}$ of deposition process, the commercial electrolyte exhibits relatively higher electrode impedance Figure S1A. At the same time, $4 \mathrm{M}$ LiODFB-DME electrolyte shows small impedance Figure S1B. 

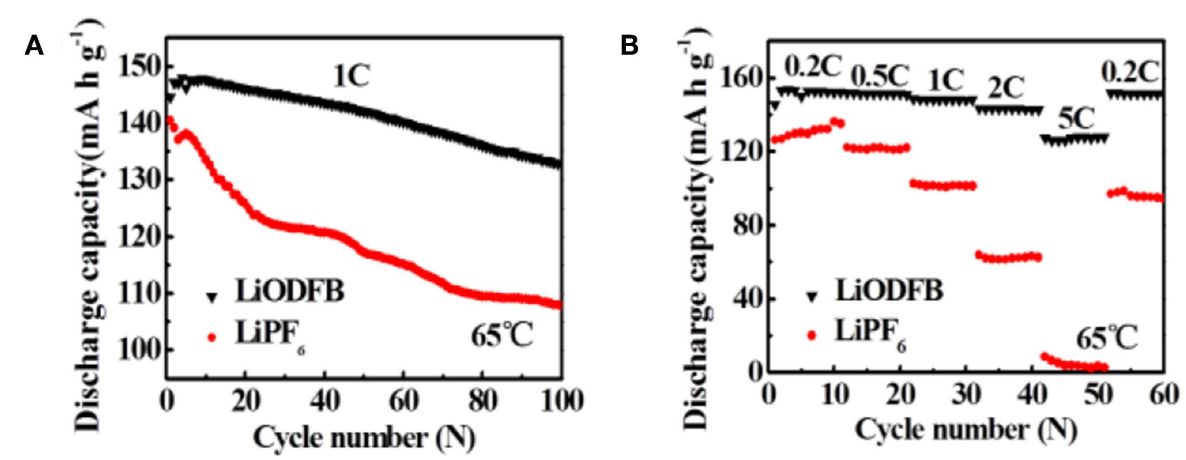

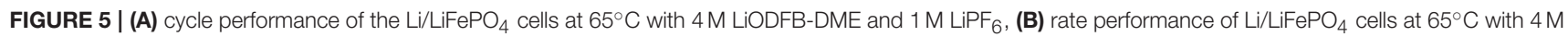
LiODFB-DME and $1 \mathrm{M} \mathrm{LiPF}_{6}$.

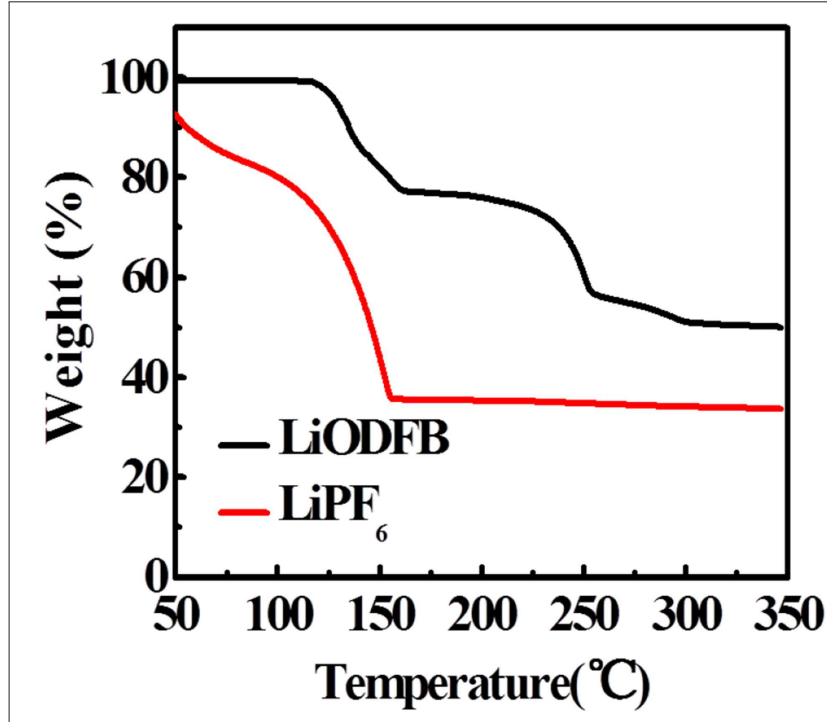

FIGURE 6 | TGA curves of the fully-charged $\mathrm{LiFePO}_{4}$ electrode cycled in LiODFB and LiPF 6 electrolytes.

The most possible reason for the small impedance is attributed to the formation of the stable SEI film, which is consistent with the results of a dense surface morphology in Figure 1b. Polarization measurements of the $\mathrm{Li} / \mathrm{Li}$ cells were conducted to understand the effect of different electrolytes on stabling the interface between the Li metal and electrolyte. During this experiment, a constant deposition/dissolution current density of $1.0 \mathrm{~mA} \mathrm{~cm}^{-2}$ was passed through the $\mathrm{Li} / \mathrm{Li}$ cells for $3,000 \mathrm{~h}$ at room temperature. The $1 \mathrm{M} \mathrm{LiPF}_{6}$ electrolyte shows the great over-voltage and an ascending tendency with time (Figure 2), which is consistent with previous report (Webb et al., 2014). Surprisingly, the $4 \mathrm{M}$ LiODFB-DME electrolyte appears excellent Li deposition/dissolution performance with the stable and low over-voltage. Polarization is lower than $1 \mathrm{M} \mathrm{LiPF}_{6}$ electrolyte. Besides, influenced by high viscosity, on the one hand, it possibly increases the pressure from the electrolyte to push back growing dendrites, resulting in a more uniform deposition on the surface of the anode. On the other hand, high viscosity limits anion convection near deposition area, which is also helpful to deposit uniformly. All of the above results confirm that the high concentration of LiODFB electrolyte in ether solvent can ensure the cycle stability in the negative.

\section{DISCUSSION}

\section{Discussion for the Possible Function Mechanism}

Figure 3A compares the $\mathrm{C} 1 \mathrm{~s}$ spectra of the delithiated graphite electrodes extracted from the cell after several cycles. The dominant peak at $284.6 \mathrm{eV}$ originates mainly from the $\mathrm{sp}^{2}$ hybridized graphite, but also includes contributions from conductive carbon added into the electrode composites. The broad feature at $286.6 \mathrm{eV}$ is assigned mainly to the $\mathrm{C}$ atoms of the $\mathrm{C}-\mathrm{O}-\mathrm{C}$ groups in the poly(ethylene oxide), $\mathrm{PEO}$, which is formed upon solvent polymerization. The formation of $\mathrm{PEO}$ is also confirmed by the peak at $533.6 \mathrm{eV}$ in the O1s XPS spectra in Figure 3B. The rather broad peaks at $288.6 \mathrm{eV}$ in Figure 4A are assigned to carbon in $\mathrm{C}=\mathrm{O}$ groups in organic $\mathrm{Li}$ alkyl carbonates $\left(\mathrm{ROCO}_{2} \mathrm{Li}\right)$ and $\mathrm{Li}$ carbonates $\left(\mathrm{Li}_{2} \mathrm{CO}_{3}\right)$, respectively. The corresponding peak of the oxygen atoms in these groups is detected in the O1s spectra (Figure 3C) at $531.8 \mathrm{eV}$. Figure 3C, the $\mathrm{F} 1 \mathrm{~s}$ peak at $687.5 \mathrm{eV}$ are assigned with PVDF, while the peak at $684.2 \mathrm{eV}$ corresponding to $\mathrm{LiF}$ is much stronger, agreeing with the inorganic inner layer of SEI. However, there is almost no difference in contrast to the case of the commercial electrolyte in the C1s, O1s, and F1s spectra. The difference appears at F1s spectra. The B1s peaks at $193.8 \mathrm{eV}$ and $192.6 \mathrm{eV}$ (Figure 3D) indicates the presence of LiODFB and material with $\mathrm{B}-\mathrm{O}$, respectively. And no such peak in commercial electrolyte.

This result indicates that the $\mathrm{B}-\mathrm{O}$ bond is involved in the formation of the film, making the SEI film is much denser (Xu et al., 2016). It is consistent with the results of a dense surface morphology in Figure 1b.

\section{The Electrochemical Performances of LiFePO ${ }_{4}$ Batteries}

The assembled $\mathrm{Li} / \mathrm{LiFePO}_{4}$ coin cells are used to compare the cycling and rate stability using $4 \mathrm{M}$ LiODFB-DME electrolyte and $1 \mathrm{M} \mathrm{LiPF}_{6}$ electrolyte at room temperature in this part. 


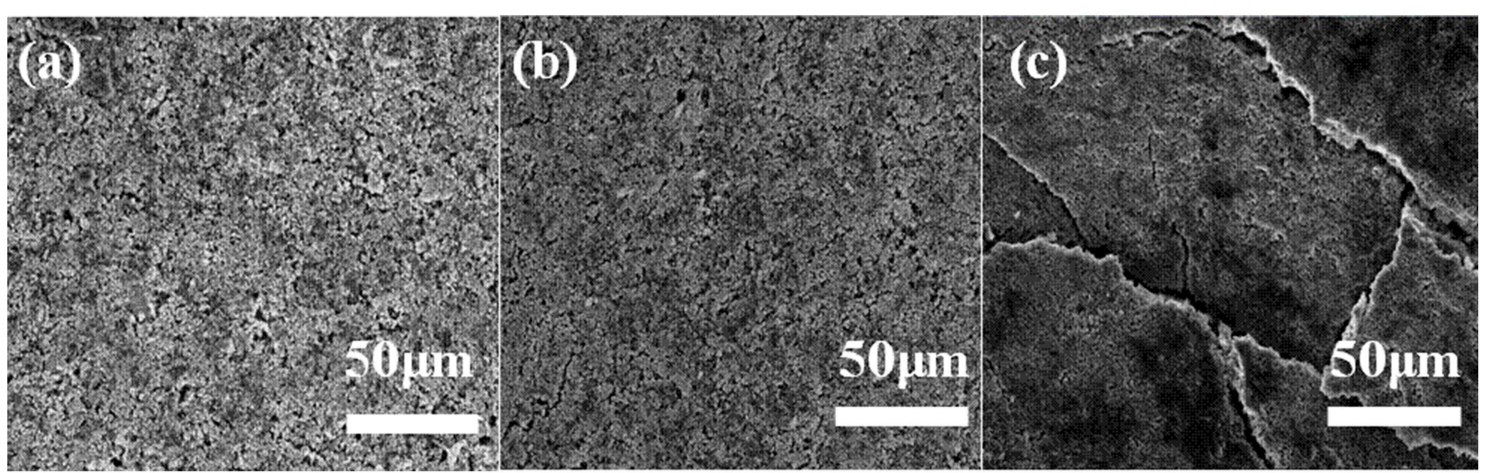

FIGURE 7 | SEM images for pristine cathode (a) cathodes after 100 cycles at $65^{\circ} \mathrm{C}$ with (b) the $4 \mathrm{M}$ LiODFB-DME; (c) the $1 \mathrm{M}$ LiPF$_{6}$.

Figure 4A shows the first charge/discharge profiles of the cells in different electrolytes at the $0.2^{\circ} \mathrm{C}$ current rate $\left(1^{\circ} \mathrm{C}=170\right.$ $\mathrm{mAh} \mathrm{g}^{-1}$ ). The cells are initially activated at $0.2^{\circ} \mathrm{C}$ for three cycles and then cycled at $0.5^{\circ} \mathrm{C}$. The cells display similar voltage curves, but the cell using the $4 \mathrm{M}$ LiODFB-DME electrolyte exhibits slightly smaller discharge capacities of $129 \mathrm{mAh} \mathrm{g}^{-1}$, while the initial discharge capacity of $1 \mathrm{M} \mathrm{LiPF}_{6}$ is $133 \mathrm{mAh}$ $\mathrm{g}^{-1}$. The result is directly related to the lower ionic conductivity of $4 \mathrm{M}$ LiODFB-DME electrolyte compared to $\mathrm{LiPF}_{6}$-based electrolyte. The long-term cycling performances of $\mathrm{Li} / \mathrm{LiFePO}_{4}$ cells are depicted in Figure 4C. There is no obvious difference after 200 cycles, and $4 \mathrm{M}$ LiODFB-DME electrolyte shows little superiority compared to commercial $\mathrm{LiPF}_{6}$-based electrolyte. However, LiODFB electrolyte shows excellent CE of $99 \%$ over 200 cycles; while the cell with the $\mathrm{LiPF}_{6}$ is only $89 \%$ after 200 cycles (Figure 4B). It shows that LiODFB salt greatly improves the cycling stability, but the delivered capacity might have been hindered by the electrolyte's undesirable ionic conductivity. Figure 4D presents the rate capability of $\mathrm{Li} / \mathrm{LiFePO}_{4}$ cell in $1 \mathrm{M}$ $\mathrm{LiPF}_{6}$ and $4 \mathrm{M}$ LiODFB-DME electrolytes. Discharge capacity of cells with $4 \mathrm{M}$ LiODFB-DME are $154.8 \mathrm{mAh} \mathrm{g}^{-1}$ at $0.2^{\circ} \mathrm{C}$, $143.3 \mathrm{mAh} \mathrm{g}^{-1}$ at $0.5^{\circ} \mathrm{C}, 134.4 \mathrm{mAh} \mathrm{g}^{-1}$ at $1.0^{\circ} \mathrm{C}, 123.1 \mathrm{mAh}$ $\mathrm{g}^{-1}$ at $2.0^{\circ} \mathrm{C}$, and $99.5 \mathrm{mAh} \mathrm{g}^{-1}$ at $5.0^{\circ} \mathrm{C}$. As comparison, discharge capacity of cells with $1 \mathrm{M} \mathrm{LiPF}_{6}$ are $143.9 \mathrm{mAh} \mathrm{g}$ at $0.2^{\circ} \mathrm{C}, 136.7 \mathrm{mAh} \mathrm{g}^{-1}$ at $0.5^{\circ} \mathrm{C}, 128.3 \mathrm{mAh} \mathrm{g}^{-1}$ at $1.0^{\circ} \mathrm{C}$, $118.9 \mathrm{mAh} \mathrm{g}^{-1}$ at $2.0^{\circ} \mathrm{C}$, and $95.8 \mathrm{mAh} \mathrm{g}^{-1}$ at $5.0^{\circ} \mathrm{C}$. Voltage profiles of both cells are also very similar Figure S2. It seems that discharged capacity at different cycling rates of cells with $4 \mathrm{M}$ LiODFB-DME electrolyte are slightly higher than cells with $1 \mathrm{M} \mathrm{LiPF}_{6}$ electrolyte. The outstanding thermal stability and water stability LiODFB leads to very weak side reactions and the dissolution of iron is suppressed. These are the reasons of better cycling performance of cells in $4 \mathrm{M}$ LiODFB-DME electrolyte. All in all, the electrochemical performances of the two electrolytes capacity reach a considerable level at room temperature. These also can be confirmed on the impedance test results in Figure S2.

Furthermore, the cycling performances of $\mathrm{Li} / \mathrm{LiFePO}_{4}$ cells at $65^{\circ} \mathrm{C}$ are assessed in both electrolytes (Figure 5A). In Figure 5A, when the cell is working in $\mathrm{LiPF}_{6}$ electrolyte, the capacity decay rapidly after cycles. We can discover it only has capacity retention of $79.3 \%$ after 100 cycles, which is consistent with the result reported by ZHANG et al. As a comparison, excellent cycle stability is observed when using $4 \mathrm{M}$ LiODFB-DME electrolyte, with high capacity retention of $92.5 \%$ after 100 cycles. The rate capability of $\mathrm{Li} / \mathrm{LiFePO}_{4}$ at $65^{\circ} \mathrm{C}$ in different electrolytes is obtained in Figure 5B. Much larger discharge capacities are showed at higher rates for the $4 \mathrm{M}$ LiODFB-DME. For example, the $\mathrm{LiPF}_{6}$ electrolyte shows discharge capacities of 101.1 and $62.5 \mathrm{mAh} \mathrm{g}^{-1}$ at the 1.0 and $2.0^{\circ} \mathrm{C}$ rates, respectively, even the capacities of cell is almost close to zero at $5.0^{\circ} \mathrm{C}$; While these values are increased to $148.1,142.8,127.7 \mathrm{mAh} \mathrm{g}^{-1}$ at the $1.0,2.0$, and $5.0^{\circ} \mathrm{C}$ rates for the LiODFB electrolyte. This demonstrates that LiODFB not only improve the cycling stability but also improve the rate capability in high temperature. It seems that the better thermal stability of LiODFB salts plays an important role in improving the high-temperature resilience of $\mathrm{LiFePO}_{4}$ electrode.

We used TGA to study the thermal stability of fully charged electrodes in electrolytes (Figure 6). Evaporation of the adsorbed organic solvent appears as their weight loss before $150^{\circ} \mathrm{C}$ in $4 \mathrm{M}$ LiODFB-DME electrolyte. As the heating progressed, a significant weight loss begins to appear at about $250^{\circ} \mathrm{C}$, which originates from the decomposition of the SEI film. In contrast, the large-scale weight decay starts at $50^{\circ} \mathrm{C}$ in $1 \mathrm{M} \mathrm{LiPF}_{6}$, and the mass attenuation has reached a minimum at $150^{\circ} \mathrm{C}$, proving that the SEI film has completely broken before $150^{\circ} \mathrm{C}$. It shows that $4 \mathrm{M}$ LiODFB-DME electrolyte produces a more stable SEI film and establishes a more gentle interface between the electrode and the electrolyte. So the fully delithiated electrode is protected from directly contacting with the electrolyte. Therefore, the LiODFB electrolyte improves the thermal stability of the highly oxidized charged electrode.

\section{SEM Micrographs of $\mathrm{LiFePO}_{4}$ Electrodes}

SEM analysis is performed to illustrate electrode images before and after 100 cycles in both electrolytes at $65^{\circ} \mathrm{C}$. The $\mathrm{LiFePO}_{4}$ electrode after long-term cycling in $4 \mathrm{M}$ LiODFBDME electrolyte (Figure 7b) shows a similar morphology to the pristine $\mathrm{LiFePO}_{4}$ electrode (Figure 7a). In comparison, the electrode is severely eroded in $1 \mathrm{M} \mathrm{LiPF}_{6}$ electrolyte after 
100 cycles at $65^{\circ} \mathrm{C}$ in Figure $7 \mathrm{c}$, and we can found that the $\mathrm{LiFePO}_{4}$ electrode surface in $1 \mathrm{M} \mathrm{LiPF}_{6}$ electrolyte displays some cracks among the particles. It is result from the poor thermal stability of the $1 \mathrm{M} \mathrm{LiPF}_{6}$ electrolyte. The decomposition of $\mathrm{LiPF}_{6}$ in high temperature causes serious capacity decay rapidly, resulting in very poor electrochemical performance, as shown in Figure 5A. The higher the current density, this effect is more serious in Figure 5B. These SEM observations confirm that $4 \mathrm{M}$ LiODFB-DME electrolyte has excellent compatibility with $\mathrm{LiFePO}_{4}$ cathodes during battery cycling at high temperature Figure S3 shows the EDS layered image of $\mathrm{LiFePO}_{4}$ electrode after 100 cycles at high magnification. The surface of the $\mathrm{LiFePO}_{4}$ electrode consists of five main elements: O, P, F, and Fe. The Fe and $\mathrm{P}$ elements are mainly derived from $\mathrm{LiFePO}_{4}$ electrode and the $\mathrm{O}, \mathrm{F}$, and $\mathrm{B}$ elements are derived from LiODFB lithium salt. It is proved that the $\mathrm{B}$ element from the LiODFB-based electrolyte participates the formation of the surface film of $\mathrm{LiFePO}_{4}$ cathode. And the participation of the B elements makes the surface film of $\mathrm{LiFePO}_{4}$ cathode smooth and compact. All in all, the LiODFB lithium salt protects the cathode. In addition, the SEM images of the morphologies of $\mathrm{Al}$ foils is shown in Figure S4. The surface of the Al foils had a large difference. The concentrated electrolytes suppress the $\mathrm{Al}$ corrosion more effectively.

\section{CONCLUSIONS}

In this work, we first developed a concentrated electrolyte based on LiODFB in ether solvents for LMBs. Based on $4 \mathrm{M}$ LiODFB-DME electrolyte, Li was deposited on the copper foil and its surface showed smooth solid particle morphology without any dendrites. For the $\mathrm{Li} / \mathrm{LiFePO}_{4}$ cells, it is demonstrated that $4 \mathrm{M}$ LiODFB-DME electrolyte exhibit good electrochemical performance, especially at the elevated temperature. $\mathrm{Li} / \mathrm{LiFePO}_{4}$

\section{REFERENCES}

Abouimrane, A., Ding, J., and Davidson, I. J. (2009). Liquid electrolyte based on lithium bis-fluorosulfonyl imide salt: Al corrosion studies and lithium ion battery investigations. J. Power Sources 189, 693-696. doi: 10.1016/j.jpowsour.2008.08.077

Aurbach, D., Gamolsky, K., Markovsky, B., Gofer, Y., Schmidt, M., Heider, U., et al. (2003). On the use of vinylene carbonate (VC) as an additive to electrolyte solutions for Li-ion batteries. Electrochim. Acta 47, 1423-1439. doi: 10.1016/S0013-4686(01) 00858-1

Bian, X., Ge, S., Pang, Q., Zhu, K., Wei, Y., Zou, B., et al. (2017). A novel lithium difluoro(oxalate) borate and lithium hexafluoride phosphate dualsalt electrolyte for Li-excess layered cathode material. J. Alloy. Compd. 736, 136-142. doi: 10.1016/j.jallcom.2017.11.126

Cekic-Laskovic, I., von Aspern, N., Imholt, L., Kaymaksiz, S., Oldiges, K., Rad, B. R., et al. (2017). Synergistic effect of blended components in nonaqueous electrolytes for lithium ion batteries. Top. Curr. Chem. 375, 37-100. doi: 10.1007/s41061-017-0125-8

Cheng, X. B., Hou, T. Z., Zhang, R., Peng, H. J., Zhao, C. Z., Huang, J. Q., et al. (2016). Dendrite-free lithium deposition induced by uniformly distributed lithium ions for efficient lithium metal batteries. Adv. Mater. 28, 2888-2895. doi: $10.1002 /$ adma.201506124 cells using $4 \mathrm{M}$ LiODFB-DME electrolyte show a good capacity retention $(92.5 \%)$ at elevated temperature $\left(65^{\circ} \mathrm{C}\right)$, which is much higher than that of $1 \mathrm{M} \mathrm{LiPF} 6$ electrolyte (79.2\%). This research offers the possibility of rapid industrialization of LMBs.

\section{DATA AVAILABILITY}

The raw data supporting the conclusions of this manuscript will be made available by the authors, without undue reservation, to any qualified researcher.

\section{AUTHOR CONTRIBUTIONS}

ZF and JY conceived and designed the experiments. NG, JP, XL, and NM performed the experiments. NG, CS, and XL analyzed the data. NG and NM wrote the manuscript. KX designed the scheme. All authors reviewed the manuscript and approved the final version.

\section{ACKNOWLEDGMENTS}

We acknowledge the financial support of this work by the Project (51574191, 51304151) supported by the National Natural Science Foundation of China; Project (18JK0474) supported by Shaanxi Provincial Education Department, China; Project (2018JM5135) supported by the Natural Science Basic Research Plan in Shaanxi Province, China.

\section{SUPPLEMENTARY MATERIAL}

The Supplementary Material for this article can be found online at: https://www.frontiersin.org/articles/10.3389/fchem. 2019.00494/full\#supplementary-material

Etacheri, V., Marom, R., Elazari, R., Salitra, G., and Aurbach, D. (2011). Challenges in the development of advanced Li-ion batteries: a review. Energy Environ. Sci. 4, 3243-3262. doi: 10.1039/C1EE01598B

Goodenough, J. B., and Kim, Y. (2014). Challenges for rechargeable li batteries. Chem. Mater. 22, 587-603. doi: 10.1021/cm901452z

Jung, H., Park, S. H., Jeon, J., Choi, Y., Yoon, S., Cho, J. J., et al. (2013). Fluoropropane sultone as an SEI-forming additive that outperforms vinylene carbonate. J. Mater. Chem. 1, 11975-11981. doi: 10.1039/C3TA12580G

Kozen, A. C., Lin, C. F., Pearse, A. J., Schroeder, M. A., Han, X., Hu, L., et al. (2015). Next-generation lithium metal anode engineering via atomic layer deposition. ACS Nano 9, 5884-5892. doi: 10.1021/acsnano.5b02166

Li, F., Gong, Y., Jia, G., Wang, Q., Peng, Z., Fan, W., et al. (2015). A novel dual-salts of LiTFSI and $\mathrm{LiODFB}$ in $\mathrm{LiFePO}_{4}$-based batteries for suppressing $\mathrm{Al}$ corrosion and improving cycling stability. J. Power Sources 295, 47-54. doi: 10.1016/j.jpowsour.2015.06.117

Li, L., Zhou, S., Han, H., Li, H., Nie, J., Armand, M., et al. (2011). Transport and electrochemical properties and spectral features of non-aqueous electrolytes containing LiFSI in linear carbonate solvents. J. Electrochem. Soc. A 158, 74-82. doi: 10.1149/2.jes120006

Liang, Z., Lin, D., Zhao, J., Lu, Z., Liu, Y., Liu, C., et al. (2016). Composite lithium metal anode by melt infusion of lithium into a 3D conducting scaffold with lithiophilic coating. Proc. Natl. Acad. Sci. U.S.A. 113, 2862-2867. doi: $10.1073 /$ pnas. 1518188113 
Liang, Z., Zheng, G., Liu, C., Liu, N., Li, W., Yan, K., et al. (2015). Polymer nanofiber-guided uniform lithium deposition for battery electrodes. Nano Lett. 152910-2916. doi: 10.1021/nl5046318

Liu, J., Chen, Z., Busking, S., and Amine, K. (2007). Lithium difluoro(oxalato)borate as a functional additive for lithium-ion batteries. Electrochem. Commun. 9, 475-479. doi: 10.1016/j.elecom.2006.10.022

Park, K., Yu, S., Lee, C., and Lee, H. (2015). Comparative study on lithium borates as corrosion inhibitors of $\mathrm{Al}$ current collector in lithium bis(fluorosulfonyl)imide electrolytes. J. Power Sources 296, 197-203. doi: 10.1016/j.jpowsour.2015.07.052

Placke, T., Kloepsch, R., Dühnen, S., and Winter, M. (2017). Lithium ion, lithium metal, and alternative rechargeable battery technologies: the odyssey for high energy density. J. Solid State Electrochem. 21, 1939-1964. doi: 10.1007/s10008-017-3610-7

Poyraz, A. S., Laughlin, J., and Zec, Z. (2019). Improving the cycle life of cryptomelane type manganese dioxides in aqueous rechargeable zinc ion batteries: the effect of electrolyte concentration. Electrochim. Acta 305,423-432. doi: 10.1016/j.electacta.2019.03.093

Pritzl, D., Solchenbach, S., Wetjen, M., and Gasteiger, H. A. (2017). Analysis of vinylene carbonate (VC) as additive in graphite/LiNi ${ }_{5}{ }_{5} \mathrm{Mn}_{1.5} \mathrm{O}_{4}$ cells. $J$. Electrochem. Soc. A 164, 2625-2635. doi: 10.1149/2.1441712jes

Qian, J., Henderson, W. A., Xu, W., Bhattacharya, P., Engelhard, M., Borodin, O., et al. (2015). High rate and stable cycling of lithium metal anode. Nat. Commun. 6, 6362-6370. doi: 10.1038/ncomms7362

Qian, Y., Schultz, C., Niehoff, P., Schwieters, T., Nowak, S., Winter, M., et al. (2016). Investigations on the electrochemical decomposition of the electrolyte additive vinylene carbonate in Li metal half cells and lithium ion full cells. J. Power Sources 332, 60-71. doi: 10.1016/j.jpowsour.2016.09.100

Shi, L., Xu, A., and Zhao, T. (2017). First-principles investigations of the working mechanism of $2 \mathrm{D}$ h-BN as an interfacial layer for the anode of lithium metal batteries. ACS Appl. Mater. Inter. 9, 1987-1994. doi: 10.1021/acsami.6b14560

Suo, L., Hu, Y. S., Li, H., Armand, M., and Chen, L. (2013). A new class of Solventin-Salt electrolyte for high-energy rechargeable metallic lithium batteries. Nat. Commun. 4, 1481-1489. doi: 10.1038/ncomms2513

Thompson, R. S., Schroeder, D. J., Lopez, C. M., Neuhold, S., and Vaughey, J. T. (2011). Stabilization of lithium metal anodes using silane-based coatings. Electrochem. Commun. 13, 1369-1372. doi: 10.1016/j.elecom.2011.08.012

Webb, S. A., Baggetto, L., Bridges, C. A., and Veith, G. M. (2014). The electrochemical reactions of pure indium with $\mathrm{Li}$ and $\mathrm{Na}$ : anomalous electrolyte decomposition, benefits of FEC additive, phase transitions and electrode performance. J. Power Sources 248, 1105-1117. doi: 10.1016/j.jpowsour.2013.10.033

Wu, F., Ye, Y., Chen, R., Qian, J., Zhao, T., Li, L., et al. (2015). A systematic effect for an ultra-long cycle lithium-sulfur battery. Nano Lett. 15, 7431-7439. doi: 10.1021/acs.nanolett.5b02864

Wu, Q., Lu, W., Miranda, M., Honaker-Schroeder, T. K., Lakhsassi, K. Y., Dees, D., et al. (2012). Effects of lithium difluoro(oxalate)borate on the performance of Li-rich composite cathode in Li-ion battery. Electrochem. Commun. 24, 78-81. doi: 10.1016/j.elecom.2012.08.016

Xie, K., Wei, W., Yuan, K., Lu, W., Guo, M., Li, Z., et al. (2016). Toward dendrite-free lithium deposition via structural and interfacial synergistic effects of 3D graphene@Ni scaffold. ACS Appl. Mater. Interfaces 8, 26091-26097. doi: 10.1021/acsami.6b09031

Xie, K., Yuan, K., Zhang, K., Shen, C., Lv, W., Liu, X., et al. (2017). Dual functionalities of carbon nanotube films for dendrite-free and high energy- high power lithium-sulfur batteries. ACS Appl. Mater. Interfaces 9, 4605-4613. doi: 10.1021/acsami.6b14039

Xu, Y., Liu, J., Zhou, L., Zeng, L., and Yang, Z. (2017). FEC as the additive of $5 \mathrm{~V}$ electrolyte and its electrochemical performance for $\mathrm{LiNi}_{0.5} \mathrm{Mn}_{1.5} \mathrm{O}_{4}$. J. Electroanal. Chem. 791, 109-116. doi: 10.1016/j.jelechem.2017.03.017

Xu, Z., Wang, J., Yang, J., Miao, X., Chen, R., Qian, J., et al. (2016). Enhanced performance of a lithium-sulfur battery using a carbonate-based electrolyte. Angew. Chem. Int. Ed. 55, 10372-10375. doi: 10.1002/anie.201605931

Yamada, Y., Wang, J., Ko, S., Watanabe, E., and Yamada, A. (2019). Advances and issues in developing salt-concentrated battery electrolytes. Nat. Nanotechnol. 14, 200-207. doi: 10.1038/s41560-019-0336-Z

Yang, C. P., Yin, Y. X., Zhang, S. F., Li, N. W., and Guo, Y. G. (2015) Accommodating lithium into 3D current collectors with a submicron skeleton towards long-life lithium metal anodes. Nat. Commun. 6, 8058. doi: 10.1038/ncomms9058

Zhamu, A., Chen, G., Liu, C. G., Neff, D., Fang, Q., Yu, Z. N., et al. (2012). Reviving rechargeable lithium metal batteries: enabling next-generation high-energy and high-power cells. Energy Environ. Sci. 5, 5701-5707. doi: 10.1039/C2EE02911A

Zhang, R., Cheng, X. B., Zhao, C. Z., Peng, H. J., Shi, J. L., Huang, J. Q., et al. (2016). Conductive nanostructured scaffolds render low local current density to inhibit lithium dendrite growth. Adv. Mater. 28, 2155-2162. doi: 10.1002/adma.201504117

Zhang, S. S. (2006). An unique lithium salt for the improved electrolyte of Li-ion battery. Electrochem. Commun. 9, 1423-1428. doi: 10.1016/j.elecom.2006.06.016

Zhang, Z., Chen, X., Li, F., Lai, Y., Li, J., Liu, P., et al. (2010). $\mathrm{LiPF}_{6}$ and lithium oxalyldifluoroborate blend salts electrolyte for LiFePO 4 /artificial graphite lithium-ion cells. J. Power Sources 21, 7397-7402. doi: 10.1016/j.jpowsour.2010.05.056

Zhang, Z. A., Zhao, X. X., Peng, B., Lai, Y. Q., Zhang, Z. Y., Li, J., et al. (2015). Mixed salts for lithium iron phosphate-based batteries operated at wide temperature range. Transac. Nonferr. Metal. Soc. 25, 2260-2265. doi: 10.1016/S1003-6326(15)63839-0

Zhou, H., Liu, F., and Li, J. (2012). Preparation, thermal stability and electrochemical properties of LiODFB. J. Mater. Sci. Technol. 28, 723-727. doi: 10.1016/S1005-0302(12)60121-2

Zhou, H., Xiao, K., and Li, J. (2016). Lithium difluoro(oxalate)borate and LiBF 4 blend salts electrolyte for $\mathrm{LiNi}_{0.5} \mathrm{Mn}_{1.5} \mathrm{O}_{4}$ cathode material. J. Power Sources 302, 274-282. doi: 10.1016/j.jpowsour.2015.10.073

Zugmann, S., Moosbauer, D., Amereller, M., Schreiner, C., and Wudy, F. (2011). Electrochemical characterization of electrolytes for lithium -ion batteries based on lithium difluoromono(oxalato)borate. J. Power Sources 196 1417-1424. doi: 10.1016/j.jpowsour.2010.08.023

Conflict of Interest Statement: The authors declare that the research was conducted in the absence of any commercial or financial relationships that could be construed as a potential conflict of interest.

Copyright (C) 2019 Yu, Gao, Peng, Ma, Liu, Shen, Xie and Fang. This is an open-access article distributed under the terms of the Creative Commons Attribution License (CC $B Y)$. The use, distribution or reproduction in other forums is permitted, provided the original author(s) and the copyright owner(s) are credited and that the original publication in this journal is cited, in accordance with accepted academic practice. No use, distribution or reproduction is permitted which does not comply with these terms. 